Copyright (C) 2014 IEEE. Personal use of this material is permitted. Permission from IEEE must be obtained for all other uses, in any current or future media, including reprinting/republishing this material for advertising or promotional purposes, creating new collective works, for resale or redistribution to servers or lists, or reuse of any copyrighted component of this work in other works. 


\title{
Coordination of Single-Phase Rooftop PVs to Regulate Voltage Profiles of Unbalanced Residential Feeders
}

\author{
Nelly Safitri, Farhad Shahnia and Mohammad A.S. Masoum \\ Department of Electrical and Computer Engineering \\ Curtin University \\ Perth, Australia \\ nellysafitri@postgrad.curtin.edu.au
}

\begin{abstract}
Installation of single-phase rooftop Photovoltaic (PV) systems in low voltage distribution networks is gaining increasing popularity in many countries including Australia. Utilization of rooftop PVs in residential feeders without controlling their ratings and locations may deteriorate the overall grid performance including power flows, losses and voltage profiles. This paper investigates the effectiveness and limitations of two different methods for regulating the voltage profile at low voltage residential feeders with single-phase rooftop PVs. These methods are based on the availability of voltmeters at each phase at each bus along the low voltage feeder which transmit their measurements to the controllers of the PV inverters. The main objective is to regulate the voltage profiles and reduce the voltage unbalance using drool control. The algorithm considers reactive power exchange and active power curtailment of the single-phase rooftop PVs. MATLAB-based simulation results demonstrate effectiveness of the proposed approach.
\end{abstract}

Keywords-Rooftop PV, active power curtailment, droop Control, reactive power exchange and residential feeder.

\section{INTRODUCTION}

Most low voltage distribution networks were constructed a few decades ago and are reaching their capacity limits due to the natural load growth. On the other hand, customer load demand and power quality expectations have increased considerably in recent decades along with a rapid growth in the digital technology. When the large single-phase loads and rooftop Photovoltaic (PV) systems are used, although voltages are usually well balanced at the supply side, they can become unbalanced at the customer level due to the unequal distribution of single-phase loads and PVs [1]. The high number of rooftop PVs can also change the direction of power flow and lead to voltage rise along the feeder. In addition, most of the residential rooftop PV systems are singlephase units and their integrations into the three-phase networks might also cause unbalance issues due to their random locations and ratings [2-3]. These are some of the main issues and challenges that most electrical utilities are currently encountering.

Several studies have been conducted to control and mitigate the above-mentioned voltage issues. In [4], custom power devices such as dynamic voltage restorer (DVR) and distribution static compensator (DSTATCOM) are used for voltage correction within the network and it is shown that for voltage profile and voltage unbalance improvements, DSTATCOMs are more efficient than DVRs. Reference [5] has investigated the voltage unbalance issue at different locations along the LV feeders populated with rooftop PVs based on a Monte Carlo method. This research indicates that rooftop PV installations will have minor effect on the voltage unbalance at the beginning of a LV feeder designed with engineering judgments; however, the voltage unbal- ance might increase at the end of the feeder to more than the standard limit.

Reference [6] has analyzed the voltage variation sensitivity due to PV power fluctuation in unbalanced line configuration and phase loading levels in the network. This research is carried out based on many factors such as upstream sources, phase loads, PV power fluctuation, distribution line length, phase sequences, line geometries and conductor types. Reference [7] shows that rooftop PV inverters, when controlled in droop reactive power mode, can lead to improving the voltage profile in a low voltage feeder. In this method, the PV inverters control their output voltages to a fixed value by exchanging reactive power with the feeder. However, this research only focuses on threephase PVs. Reference [8] utilizes the droop-based active power curtailment to prevent overvoltage conditions in radial low voltage feeders. Reference [9] introduces a method that states a small signal can be modelled in the active droop control method of a two-loop feedback control system (designed for the active droop control) to achieve high efficiency transient response.

This paper investigates the possibility of two methods based on distributed reactive power control and active power curtailment by single-phase rooftop PVs in three-phase unbalanced low voltage residential feeders. It is assumed that proper voltage monitoring and transmitting devices are available throughout the feeders to provide data transfer among the controllers of the rooftop PV inverters. The main objective of this research is to regulate the voltage profile and reduce the voltage unbalance. The voltage regulation results are shown for a sample network based on MATLAB simulation results. Through the simulation results the effectiveness and limitations of these methods are discussed.

\section{PRoblem Formulation AND Proposed SOlution}

Rooftop PVs inject active power during the day while most household loads are at their nominal levels. This can result in active power flow in the opposite direction toward the grid causing voltage rises beyond the acceptable levels at some nodes. On the other hand, unequal distribution of rooftop PVs will result in different voltage rises in the three phases of the feeder.

The injected reactive power and curtailed active power approaches can be applied for voltage amplitude control within the network. Recent studies have only considered three-phase PV systems; however, the situation can be very different when single-phase PVs are installed unequally at different phases and buses throughout the network and have different ratings. This paper discusses the possibility of utilizing distributed reactive power support and active power curtailment by rooftop PVs in order to regulate the network voltage profile. 


\section{A. Network Under Consideration}

The selected test network is an $11 \mathrm{kV}$ three-phase medium voltage feeder supplying a $415 \mathrm{~V}$ three-phase fourwire low voltage residential feeder (Fig. 1). This network topology is frequent in many countries including Australia [10]. The residential feeder is assumed to be unbalanced due to the distribution of loads and unequal distribution of single-phase rooftop PVs with different ratings. The network data is given in Table A1 in Appendix A.

\section{B. Network Modelling and Analysis}

An unbalanced sweep forward-backward load flow method is developed in MATLAB and used for the analysis of the three-phase four-wire radial network under consideration. The load flow calculates bus voltages along the feeder. This method is discussed in detail in Appendix B.

\section{Proposed Smart-Grid Based Technique}

Consider the LV feeder of Fig. 1 with 10 buses where each node may have single-phase PVs. Currently, based on IEEE recommended practice for utility interface of PV systems [11], the PV inverters operate in constant output power mode. Under such conditions, they only inject current with unity power factor and do not affect the voltage at their point of common coupling (PCC). If the invetres are operated in voltage control mode, each PV can correct its own PCC voltage to a desired value by injecting or absorbing the required amount of reactive power $\left(Q_{P V}\right.$,ref $)$. To minimiz PCC voltage error from its reference, each PV inverter needs to exchange reactive power with the feeder to keep the voltage of its output equal to the desired value based on the following droop control strategy:

$Q_{P V, \text { ref }}=m\left(V_{P C C, \text { ref }}-V_{P C C}\right)$

where $m$ is a coefficient and will be assigned by the reactive power-voltage $(Q-V)$ droop controller. The $Q-V$ droop controller improves the dynamic oscillations between the reactive power and the voltage variation in the system. The droop control concept is shown in Fig. 2. It is to be noted that the for rooftop PV inverters, the active powerfrequency $(P-F)$ droop controller will not be used since frequency variation is very small in the stiff grid-connected distribution networks. The $Q_{P V, \text { ref }}$ calculated in (1) must be within the inverter capacity as

$-\sqrt{S_{P V, \text { max }}^{2}-P_{P V}^{2}} \leq Q_{P V, \text { ref }} \leq \sqrt{S_{P V, \text { max }}^{2}-P_{P V}^{2}}$

where $S_{P V, \text { max }}$ is the maximum apparent power of the PV inverter. If the required $Q_{P V}$,ref is beyond its maximum injection or absorption capability, it will run on the maximum limits.

The proposed algorithm in this paper will define and calculate $V_{P C C \text {,ref }}$ based on the following conditions:

1 - If a PV is available on all three phases of bus $i, V_{P C C, r e f}$ at this bus is equal to the average of the voltage magnitudes of the three phases, i.e.

$V_{P C C, \text { ref }, i}=\frac{1}{3}\left(V_{a, i}+V_{b, i}+V_{c, i}\right)$

2- If a PV is available only on two phases of node $i$ (e.g. on phases $\mathrm{b}$ and $\mathrm{c}), V_{P C C, r e f}$ at this bus is equal to the voltage magnitude of the third phase (e.g. phase a).
3- If a PV is available only on one phase of bus $i$ (e.g. on phase a), $V_{P C C \text {,ref }}$ at bus $i$ is equal to the average of the voltage magnitudes of the other two phases, i.e.

$V_{P C C, \text { ref }, i}=\frac{1}{3}\left(V_{b, i}+V_{c, i}\right)$

4- If no PV is available on any of the phases of bus $i$, no $V_{P C C \text {,ref }}$ will be defined for that bus and its voltage will not be directly controlled. However, the voltage of this bus will be affected by the change of the voltages at the other buses of the feeder.

Note that for each bus with rooftop PV, $V_{P C C, r e f, i}$ will be determined based on the data transmitted from the installed voltmeters at each phase to the rooftop PV controller. The required communuication infrustrure is not discussed as it is beyond the scope of this paper. However, it is can be assumed to be based on ZigBee technolog which is a low bandwidth, cost and loss telecommunication device and is well suited for PV applications.

The reactive power support is an efficient and recogzized apparch to control the voltage rise or drop in the network. Another effective option to control the voltage is curtailing output active power of the rooftop PVs $\left(P_{P V}\right)$. In this method, the output active power of the PVs, dicatated by the maximum power point tracking (MPPT) algorithm $\left(P_{M P P T}\right)$ can be delibertaley reduced based on the error of the feeder voltage at a specific bus to prevent voltage rise or high voltage unbalance in the feeder, as

$P_{P V}=P_{M P P T}-n\left(V_{P C C, \text { ref }}-V_{P C C}\right)$

where $n$ is a coefficient that needs to be defined to minimize the difference between the magnitudes of all three phase volatges.

As a further step, these two methods can be combined together within an optimization concept to achieve better results. This combined active and reactive power control method needs an optimization technique to achieve the best results and is not considered in this paper.

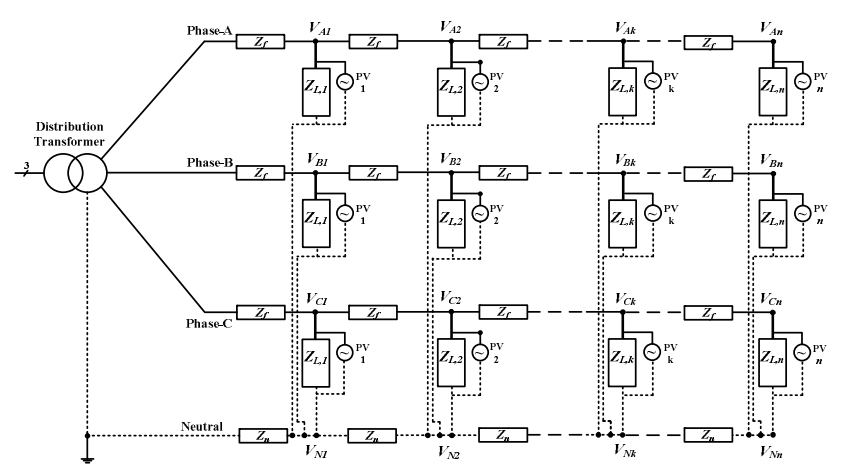

Fig. 1. Single line diagram of the simulated three-phase unbalanced residential LV network with single-phase rooftop PVs.

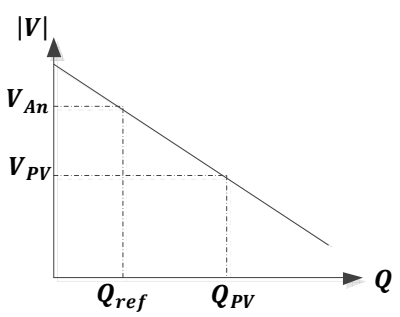

Fig. 2. The V-Q droop characteristic for rooftop PVs. 


\section{SIMULATION RESULTS}

For the network shown in Fig. 1 with the data provided in Table A1, assuming no rooftop PVs installed in the network, the three-phase voltage profile is as shown in Fig. 3.

Now, let us assume that some single-phase rooftop PVs that only generate active power have been installed in the network as given in Table A2. In this case, the three-phase voltage profile of the network is as shown in Fig. 4.

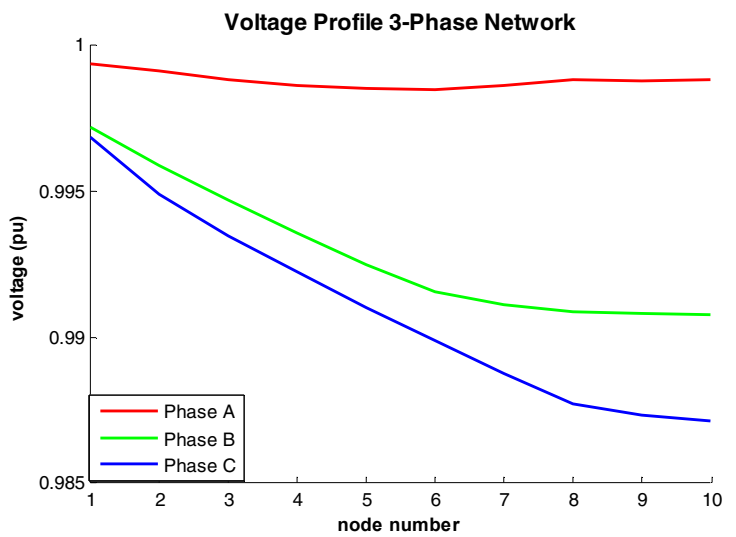

Fig. 3. Three-phase voltage profile assuming no PVs in the network.

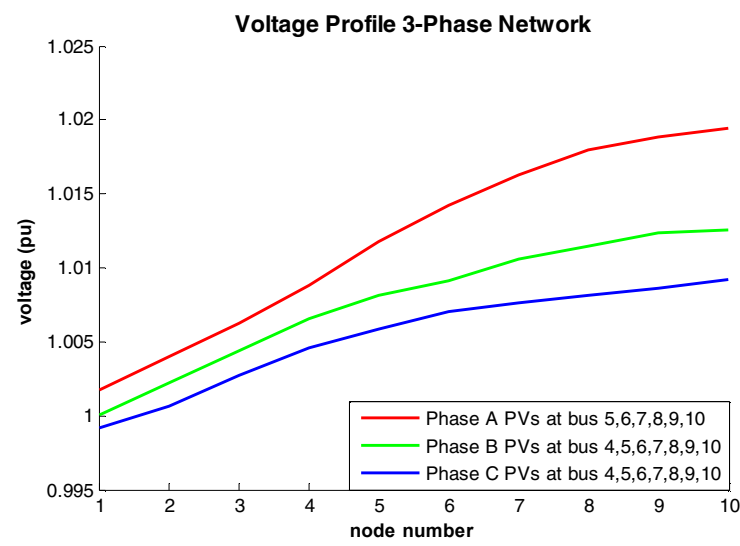

Fig. 4. Three-phase voltage profile assuming several single-phase PVs in the network.

\section{Case A-Distributed Reactive Power Support}

In this case, we assume that each PV inverter has access to the voltage magnitude of the other phases at the same bus by the help of local voltmeters and the availability of a low bandwidth communication infrastructure. Under these conditions and based on the voltage magnitude at all three phases, a desired voltage magnitude is defined for each bus. Then, PV inverter at each bus is controlled such that the desired voltage magnitude is presented at its PCC.

Figure 5 shows simulation results before and after applying the proposed voltage regulation approach. In this figure, the dashed lines represent the regulated voltage profile. Note that the three-phases have very similar voltage profiles along the feeder indicating significant reduction in voltage unbalance conditions. It is to be noted that in this case, it was assumed that 5 number of single-phase PVs are installed at buses 1,3,6,8 and 10 of phase-a, 3 number of single-phase PVs are installed at buses 3,6,9 of phase-b and 4 number of single-phase PVs are installed at buses 4,6,9 and 10 of phase-c.

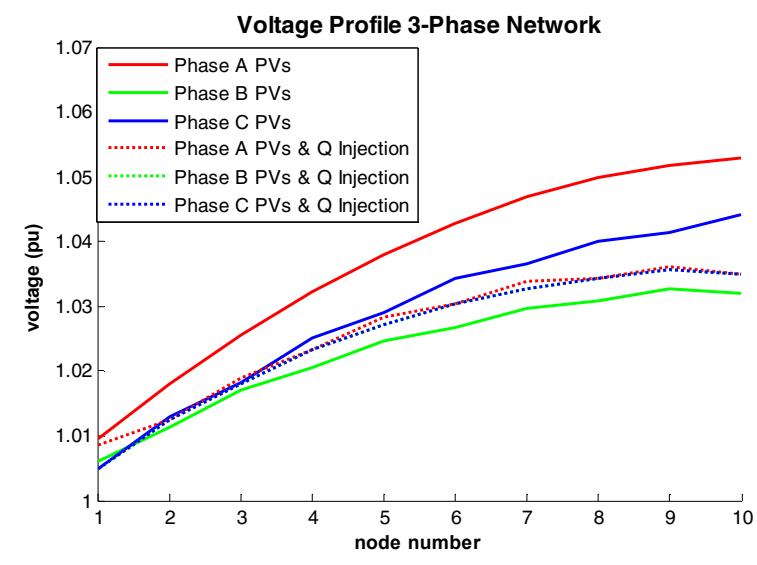

Fig. 5. Three-phase voltage profile assuming several single-phase PVs with distributed reactive power exchange capability in the network.

\section{Case B-Distributed Active Power Curtailment}

Assuming the availability of the data monitoring and transfer, as discussed earlier, let us now assume that the PV inverters are working based on only active power curtailment $\left(Q_{P V \text {,ref }}=0\right)$. Fig. 6 shows the three-phase voltage profile for a network before and after applying the active power curtailment. In this study, the coefficient of $n$ is selected as 0.7 to be most effective.

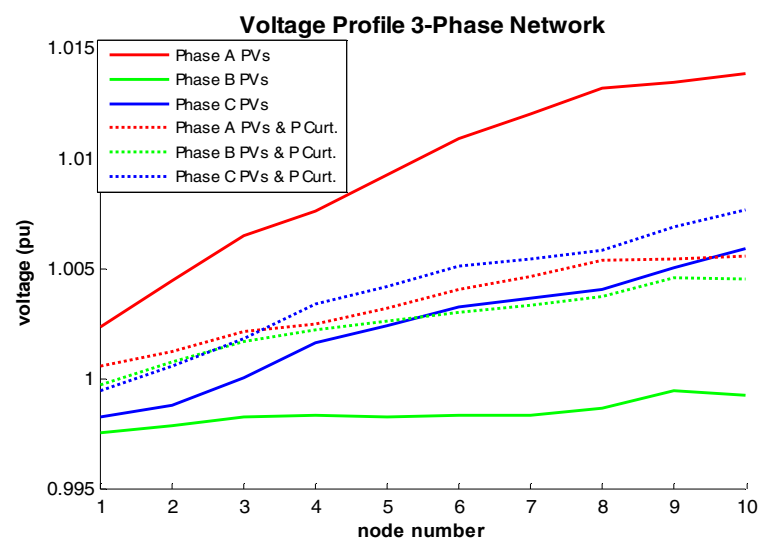

Fig. 6. Three-phase voltage profile assuming several single-phase PVs with distributed active power curtailment in the network.

\section{LIMITATIONS OF THE PROPOSED SOLUTION}

Although Fig. 5 shows that distributed reactive power support by the single-phase rooftop PVs is an efficient way of balancing the three-phase voltage profile in the network, this method highly depends on the number of PVs in each phase and their ratings. As an example, Fig. 7 shows the three-phase voltage profile of a network assuming 10 number of single-phase PVs are installed at all buses only at a certain phase (in this case phase-b only). It can be seen that, in this case, due to the number and ratings of the PVs, the distributed reactive power support method is not very effective in improving the voltage profile and reduction of voltage unbalance.

Similarly, the active power curtailment has also some limitations and its success highly depends on the number of PVs in each phase and their ratings. As an example, Fig. 8 shows the three-phase voltage profile assuming that 10 number of single-phase PVs are installed at all buses only at a certain phase (in this case phase-b only). It can be seen that, in this case, due to the number and ratings of the PVs, the distributed reactive power support method is not very effective in improving the voltage profile and reduction of voltage unbalance. 


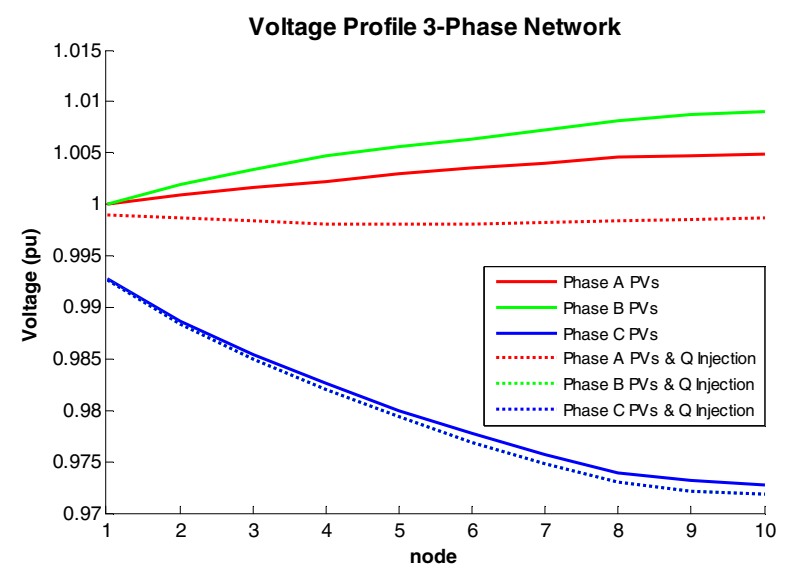

Fig. 7. Failure of reactive power exchange method in regulating voltage profile in all three phases due to unavailability of adequate number of PVs in all phases.

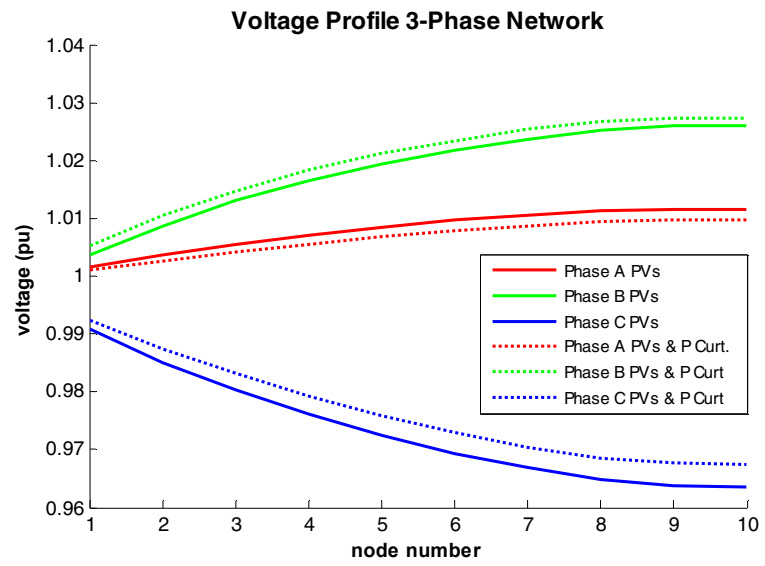

Fig. 8. Failure of active power curtailment method in regulating voltage profile in all three phases due to unavailability of adequate number of PVs in all phases.

\section{CONCLUSION}

Based on this infomartion, the controller of the PV inverters define a proper reference voltage for all threephases of the network at each bus and then the PV inverters control the voltage at that bus by exchanging reactive power with the grid. This can improve the voltage rise and voltage drop issues in the feeder. If only voltage rise problem is targeted, the curtailment of the output active power of the PVs can be a sucessful method.

The main limitation of both methods is their dependency on the number of PVs in each phase and their ratings. Through simulation results, it was shown that the methods can be sucessful or fail depending on the number of PVs on each phase and their ratings. However, the proposed methods will be successful and recommended for unbalanced three pahse networks with high pentartions of single-phase rooftop PVs.

As a future research, these two methods can be joined together and carried out as an optimization problem to minimize voltage unbalance and achieve the best voltage regulation along the feeder.

\section{APPENDIX}

\section{A. Network Data}

The network data, utilized in the simulation studies, are provided in Table A1. The ratings and installation buses of the single-phase rooftop PVs are presented in Table A2.
Table A1. Technical Parameter of the Studied LV Distribution Network

\begin{tabular}{ll}
\hline Transformer & $11 / 0.415 \mathrm{kV}, 500 \mathrm{kVA}, \Delta / \mathrm{Y}_{\text {grounded }}, \mathrm{x}_{\mathrm{tr}}=0.04 \mathrm{pu}$ \\
\hline MV Feeder & Three-phase $11 \mathrm{kV}$ radial $z=1.08+\mathrm{j} \times 0.0302 \Omega / \mathrm{km}$ \\
\hline LV Feeder & 415 V, 3-phase 4-wire, $400 \mathrm{~m}, z=0.452+\mathrm{j} \times 0.270 \Omega / \mathrm{km}$ \\
& Phase A: $\cos \varphi=0.95, z=1.28+j 2.56 \mathrm{k} \Omega$ \\
& Two loads connected to each pole \\
& Phase B: $\cos \varphi=0.95, z=1.28+j 2.56 \mathrm{k} \Omega$ \\
Load Type & Two loads connected to each pole \\
& Phase C: $\cos \varphi=0.95, z=1.28+j 2.56 \mathrm{k} \Omega$ \\
& Two loads connected to each pole
\end{tabular}

Table A2. Ratings (kW) and Installation Buses of Single-Phase Rooftop

\begin{tabular}{ccccccccccc}
\hline Node & 1 & 2 & 3 & 4 & 5 & 6 & 7 & 8 & 9 & 10 \\
\hline Phase A & 0 & 0 & 0 & 0 & 1 & 2 & 1 & 2 & 1 & 2 \\
Phase B & 0 & 0 & 0 & 1 & 2 & 1 & 2 & 1 & 2 & 1 \\
Phase C & 0 & 0 & 1 & 2 & 1 & 2 & 1 & 2 & 1 & 2 \\
\hline
\end{tabular}

\section{B. Unbalanced Load Flow for a Radial Feeder}

An unbalanced sweep forward-backward load flow [12] is considered and integrated into the developed model. The load flow calculates the bus voltages along the feeder.

For this, first, modified Carson's equations [12] are utilized for calculation of self and mutual impedance of the conductors in the $50 \mathrm{~Hz}$ system as

$$
\begin{aligned}
& Z_{i i}=r_{i}+0.04934+j 0.062832\left(\operatorname{Ln} \frac{1}{G M R_{i}}+7.10988\right) \\
& Z_{i j}=0.04934+j 0.062832\left(\operatorname{Ln} \frac{1}{D_{i j}}+7.10988\right)
\end{aligned}
$$

where $i$ and $j$ are the phase conductor (i.e. A, B, C or Neutral), $Z_{i i}$ is the self-impedance of conductor $i$ (in $\Omega / \mathrm{km}$ ), $Z_{i j}$ is the mutual impedance between two conductors $i$ and $j$ (in $\Omega / \mathrm{km}), r_{i}$ is the AC resistance of conductor $i$ (in $\Omega / \mathrm{km}$ ), $\mathrm{GMR}_{i}$ is the Geometric Mean Radius of conductor $i$ (in cm) and $D_{i j}$ is the distance between conductor $i$ and $j$ (in $\mathrm{cm}$ ). Hence, the non-transposed characteristics of the conductors, image conductors below ground and network configuration are considered in the studies. Fig. 9(a) shows the considered line configuration in this study [10]. The three-phase fourwire line segment between two adjacent buses of $k-1$ and $k$ is also shown in Fig. 9(b). From (6) and (7), the equivalent impedance for this line section is expressed as

$\left[Z_{a b c n}\right]=\left[\begin{array}{llll}Z_{a a} & Z_{a b} & Z_{a c} & Z_{a n} \\ Z_{b a} & Z_{b b} & Z_{b c} & Z_{b n} \\ Z_{c a} & Z_{c b} & Z_{c c} & Z_{c n} \\ Z_{n a} & Z_{n b} & Z_{n c} & Z_{n n}\end{array}\right]$

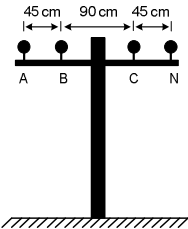

(a)

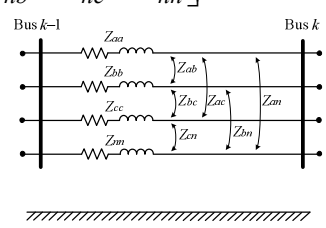

(b)
Fig 9. (a) Low voltage feeder configuration,

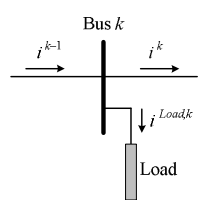

(c) (b) Impedance equivalent of a line segment between two buses,

$$
\text { (c) PQ bus model. }
$$

Assuming the transformer with a delta/star-grounded connection, which is the common distribution transformed in Australia, and using Kron reduction, (8) can be rewritten as

$$
\left[Z_{a b c}\right]=\left[\begin{array}{lll}
Z_{a a-n} & Z_{a b-n} & Z_{a c-n} \\
Z_{b a-n} & Z_{b b-n} & Z_{b c-n} \\
Z_{c a-n} & Z_{c b-n} & Z_{c c-n}
\end{array}\right]
$$


For simplicity, all calculations are carried out in per unit. Starting with a set of initial values (e.g. flat voltage set), the load currents are calculated as

$\left[I_{a b c}^{L o a d, k}\right]=\operatorname{conj}\left(\frac{\left[P_{a b c}^{L o a d, k}\right]+j\left[Q_{a b c}^{L o a d, k}\right]}{\left[V_{a b c}^{k}\right]}\right)$

where $\left[I_{a b c}{ }^{\text {Load, } k}\right]$ is a vector of three-phase load current connected to bus $k,\left[V_{a b c}{ }^{k}\right]$ is a vector of three-phase voltage of bus $k$ and $\left[P_{a b c}{ }^{\text {Load }, k}\right]$ and $\left[Q_{a b c}{ }^{\text {Load, } k}\right]$ are respectively a vector of three-phase active and reactive power consumption of the residential load connected at bus $k$.

The sum of the all load currents will flow from the first bus (transformer secondary side) to the second bus. Therefore, as shown in Fig. 9(c), the current between two adjacent buses is

$$
\left[I_{a b c}^{k}\right]=\left[I_{a b c}^{k-1}\right]-\left[I_{a b c}^{\text {Load }, k}\right]
$$

Hence, the voltage of bus $k$ can be calculated based on the voltage of bus $k-1$ in its upstream and the current passing between two buses as

$$
\left[V_{a b c}^{k}\right]=\left[V_{a b c}^{k-1}\right]-\left[Z_{a b c}\right]\left[I_{a b c}^{k}\right]
$$

Once the voltage at bus $k$ is calculated, the load current in that bus will be updated from (10) and then using (11) the current flowing from bus $k$ to $k+1$ in its downstream are updated.

Similar to the line segment, the equivalent impedance of the delta/star-grounded distribution transformer between its primary and secondary buses is expressed as

$\left[Z_{a b c}^{k}\right]=z_{t} \times I$

where $z_{t}$ is the phase impedance of the transformer and $I$ is the identity matrix. Now, the secondary-side voltage of the transformer are calculated from its primary-side voltage as [12]

$\left[V t_{a b c}^{S}\right]=[A]\left[V t_{a b c}^{P}\right]-\left[Z t_{a b c}\right]\left[I_{a b c}\right]$

where $\left[V t_{a b c}{ }^{P}\right]$ and $\left[V t_{a b c}{ }^{S}\right]$ are respectively the primary and secondary-side phase voltages of the transformer and $\left[I_{a b c}\right]$ is a vector of three-phase current passing through the transformer and

$$
[A]=\frac{1}{\sqrt{3}}\left[\begin{array}{ccc}
1 & 0 & -1 \\
-1 & 1 & 0 \\
0 & -1 & 1
\end{array}\right]
$$

\section{REFERENCES}

[1] F. Shahnia, A. Ghosh, G. Ledwich, and F. Zare, "Voltage Unbalance Reduction in Low Voltage Distribution Networks with Rooftop PVs," $20^{\text {th }}$ Australasian Universities Power Engineering Conference (AUPEC), pp. 1-5, 2010

[2] P. Trichakis, P.C. Taylor, L.M. Cipcigan, et al., "An Investigation of Voltage Unbalance in Low Voltage Distribution Networks with High Levels of SSEG," $41^{\text {st }}$ Int. Universities Power Engineering Conference (UPEC), pp. 182-186, 2006.

[3] P. Trichakis, P.C. Taylor, P.F. Lyons, and R. Hair, "Predicting the Technical Impacts of High Levels of Small-Scale Embedded Generators on Low-Voltage Networks," IET Renewable Power Generation, vol. 2, pp. 249-262, 2008.

[4] F. Shahnia, A. Ghosh, G. Ledwich, and F. Zare, "Voltage Correction in Low Voltage Distribution Networks with Rooftop PVs Using Custom Power Devices," $37^{\text {th }}$ Annual Conference on IEEE Industrial Electronics Society, pp. 991-996, 2011.

[5] F. Shahnia, R. Majumder, A. Ghosh, et al., "Sensitivity Analysis of Voltage Imbalance in Distribution Networks with Rooftop PVs," IEEE Power and Energy Society General Meeting, pp. 1-8, 2010.

[6] Y. Ruifeng and T.K. Saha, "Voltage Variation Sensitivity Analysis for Unbalanced Distribution Networks Due to Photovoltaic Power Fluctuations," IEEE Trans. on Power Systems, vol. 27, pp. 1078-1089, 2012.

[7] F. Shahnia, A. Ghosh, "Decentralized Voltage Support in a Low Voltage Feeder by Droop based Voltage Controlled PVs," $23^{\text {rd }}$ Australasian Universities Power Engineering Conference (AUPEC), pp. 1-5, 2013.

[8] R. Tonkoski, L.A.C. Lopes, and T.H.M. El-Fouly, "Droop-Based Active Power Curtailment for Overvoltage Prevention in Grid Connected PV Inverters," IEEE Int. Symp. on Industrial Electronics (ISIE), pp. 2388-2393, 2010.

[9] K. Yao, K. Lee, M. Xu, and F.C. Lee, "Optimal Design of the Active Droop Control Method for the Transient Response," $18^{\text {th }}$ IEEE Annual Applied Power Electronics Conference and Exposition (APEC), vol. 2, pp. 718-723, 2003.

[10] Distribution Construction Standards Handbook, Western Power, 2007.

[11] IEEE Recommended Practice for Utility Interface of Photovoltaic (PV) Systems, IEEE Standard 929-2000.

[12] W.H. Kersting, Distribution System Modeling and Analysis, CRC Press, 2012. 